\title{
Agenesia de arteria pulmonar derecha en un neonato. La excepcionalidad del diagnóstico en período perinatal
}

\author{
David Vallés Vallés* \\ Marta Balart** \\ María Dolores Muro** \\ Jordi Muchart* \\ Alejandro Pérez Casares*** \\ * Departamento de Diagnóstico por la Imagen. Clínica Corachan. Barcelona \\ ** Departamento de Pediatría-Neonatología. Clínica Corachan. Barcelona \\ *** Departamento de Cardiología Pediátrica. Clínica Corachan. Barcelona
}

Correspondencia

David Vallés Vallés

dvalles18@hotmail.com

\section{Recibido: 23/09/2020 \\ Aceptado:31/01/2021 \\ En línea: 30/04/2021}

Citar como: Vallés Vallés D, Balart M, Muro MD, Muchart J, Pérez Casares D. Agenesia de arteria pulmonar derecha en un neonato. La excepcionalidad del diagnóstico en período perinatal . Rev Ecocar Pract (RETIC). 2021 (Abril); 4 (1): 11-14. doi: 10.37615/retic.v4n1a6.

Cite this as: Vallés Vallés D, Balart M, Muro MD, Muchart J, Pérez Casares D. Pulmonary artery agenesis in a newborn: The exceptionality of diagnosis in the perinatal period. Rev Ecocar Pract (RETIC). 2021 (Abril); 4 (1): 11-14. doi: 10.37615/retic.v4n1a6.

\begin{tabular}{l} 
Palabras clave \\
\hline$\triangleright$ Agenesia de arteria \\
$\quad$ pulmonar \\
$\triangleright$ Cardiopatía congénita \\
$\triangleright$ Diagnóstico perinatal \\
$\triangleright$ Angiotomografía \\
$\quad$ computarizada
\end{tabular}

\section{Keywords}

$\triangleright$ Pulmonary artery agenesis

$\triangleright$ Congenital cardiac disease

$\triangleright$ Perinatal diagnosis

$\triangleright$ Computed tomography angiography

\begin{abstract}
RESUMEN
La agenesia de arteria pulmonar es un trastorno congénito que normalmente se encuentra asociado a otros defectos cardiacos congénitos, por lo que es extraño que se presente de manera aislada. Aunque puede causar síntomas durante la infancia, las anomalías aisladas de las arterias pulmonares pasan con mucha frecuencia inadvertidas hasta la adolescencia o la edad adulta temprana y, por eso, su diagnóstico en período perinatal es muy poco frecuente. Se presenta el caso de un neonato al cual se le realiza una angiotomografía computarizada (angio TC) para la confirmación del diagnóstico de agenesia de la arteria pulmonar derecha.

\begin{tabular}{l} 
ABSTRACT \\
\hline Pulmonary artery agenesis is a congenital disorder that is usually linked to other congenital cardiac diseases, being \\
infrequently found on an isolated manner. Although it can cause symptoms during childhood, pulmonary arteries \\
isolated anomalies are frequently unnoticed until adolescence or early adulthood and for this reason, its perinatal \\
diagnosis is quite unusual. A case of a newborn is presented in whom a computed tomography angiography (CTA) \\
is performed to confirm the diagnosis of right pulmonary artery agenesis.
\end{tabular}
\end{abstract}

\section{Presentación del caso}

Recién nacido varón, fruto de primera gestación a término, sin antecedentes obstétricos de interés. Nace por parto eutócico con test de Apgar 9/10 y peso al nacer de $3.050 \mathrm{~g}$. En la valoración postparto por el pediata presenta tiraje subcostal leve, taquipnea ocasional (hasta 80 respiraciones por minuto) y fatiga en las tomas por lo que requiere oxigenoterapia y, aunque no se detectan soplos cardíacos, no supera el screening cardiológico ya que presenta saturación preductal de $92-93 \%$ y postductal de $85-88 \%$.

Se solicita evaluación por cardiología pediátrica. Con 8 horas de vida se realiza una ecocardiografía transtorácica donde se identifica un tronco pulmonar de tamaño normal y una arteria pulmonar izquierda colocada en su posición habitual, pero no se visualiza la arteria pulmonar derecha en los cortes habituales (Video 1, Video 2 y Video 3). El ventrículo derecho aparece ligeramente dilatado e hipertrófico, con una insuficiencia tricuspídea ligera que permite obtener un gradiente pico de $60 \mathrm{~mm} \mathrm{Hg}$, con una válvula pulmonar normofuncionante y de tamaño normal, que sugiere elevación de las presiones en el ventrículo derecho y en la arteria pulmonar en relación con hipertensión pulmonar fisiológica en el recién nacido. El drenaje venoso pulmonar parece estar presente desde las venas derechas, aunque disminuido respecto al drenaje venoso pulmonar izquierdo. No se observan otros defectos asociados y la función cardiaca es normal.

Se realiza una radiografía de tórax donde se observa una leve hiperclaridad del pulmón derecho y un hilio pulmonar hipoplásico (Figura 1). Esto hallazgos clínico-radiológicos plantean la sospecha de agenesia de la arteria pulmonar derecha.

Con 48 horas de vida se le practica una angio TC bajo sedación. El protocolo de adquisición utilizado incluyó los siguientes parámetros: grosor de corte 1.5 mm, pitch 0.8, tiempo de rotación 0.,3 seg $80 \mathrm{Kv}, 50 \mathrm{~mA}$, grosor de reconstrucción $0,6 \mathrm{~mm}$, incremento $0.3 \mathrm{~mm}$. La administración del medio de contraste (7 ml de Omnipaque 300 mg lodo/ml) se realizó de forma manual y la adquisición se obtuvo con la ayuda de la técnica bolus tracking mediante disparo manual. La dosis total de radiación se calculó en un DLP total de 14,6 mGy/cm. En este estudio donde se obtienen imágenes torácicas con diferentes ventanas de reconstrucción. Se realizan también reconstrucciones 2D multiplanares y $3 \mathrm{D}$ en proyección de máxima intensidad (MIP) y en representación de volumen o volume rendering (VR). La angio TC confirma la agenesia de la rama pulmonar derecha con una consecuente disminución global de la vascularización del 
pulmón derecho (Figura 2). Además, las venas pulmonares superior e inferior derechas presentan un diámetro disminuido (Figura 3). Por otro lado, el tronco de la arteria pulmonar y la rama pulmonar izquierda, así como el arco aórtico y los troncos supraaórticos presentan un origen y un desarrollo normal, y no había otras anomalías en el parénquima pulmonar ni en el resto de estructuras visualizadas (Figura 4).

La evolución posterior del bebé fue favorable con normalización de la pulsioximetría y de las presiones pulmonares, buena evolución clínica. Se realizó una valoración más amplia (incluida una valoración genética), que permitió que el paciente fue dado de alta a los 8 días de vida con la indicación de evaluación periódica con el cardiólogo pediátrico. A los 4 meses de vida el paciente sigue asintomático, con buen desarrollo de peso y talla y con estimación de presiones pulmonares normales.

\section{Estudio por imagen}

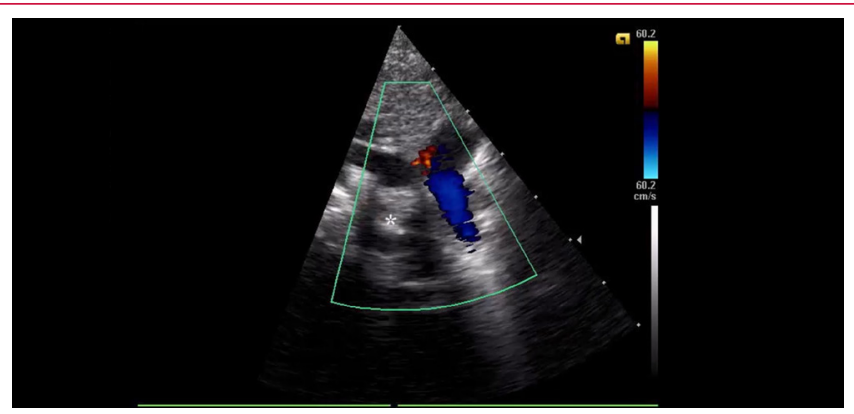

Vídeo 1. Eje corto paraesternal donde se aprecian el tronco de la arteria pulmonar y la rama pulmonar izquierda pero no se observa la rama pulmonar derecha (asterisco)

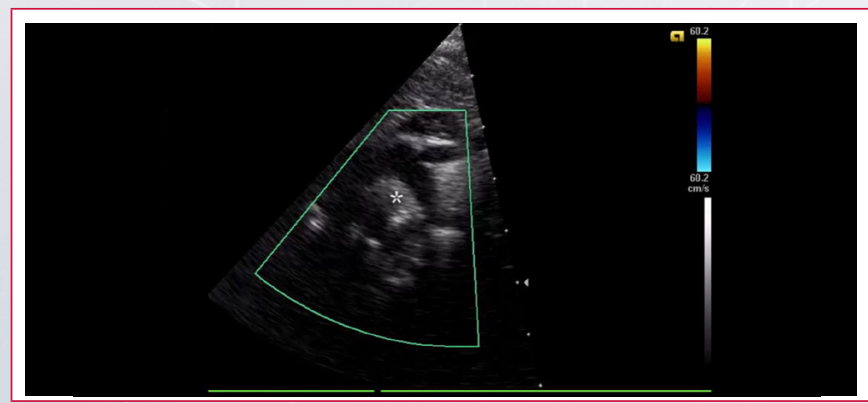

Vídeo 2. Corte supraesternal en el cual destaca la ausencia de la rama pulmonar derecha en su posición habitual (asterisco)

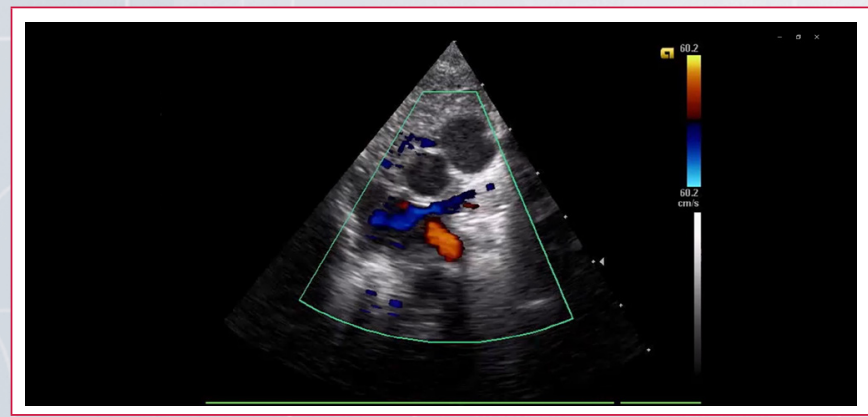

Vídeo 3. Eje corto infraclavicular en el cual se observa la llegada de flujo a la aurícula izquierda a través de las venas pulmonares izquierdas (vena pulmonar superior izquierda en azul y vena pulmonar inferior izquierda en rojo) pero no a través de las venas pulmonares derechas

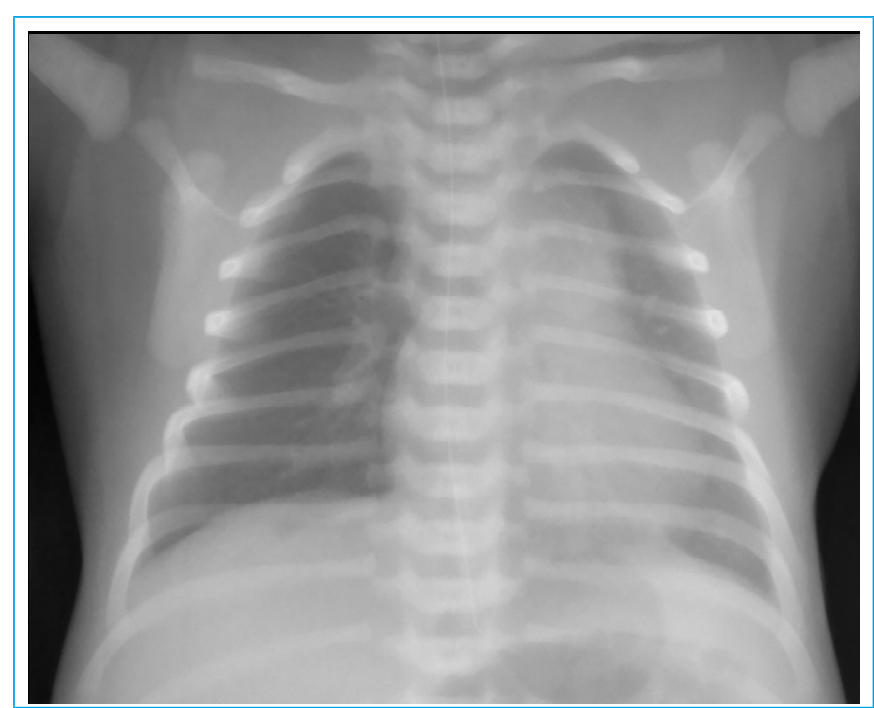

Figura 1. Radiografía de tórax que muestra leve hiperclaridad del pulmón derecho y un hilio pulmonar hipoplásico

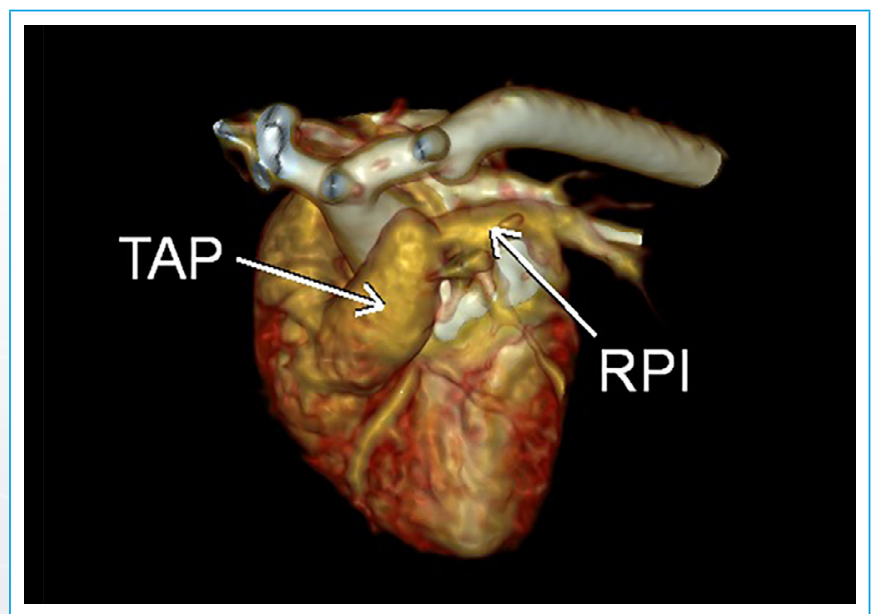

Figura 2. Reconstrucción 3D con la técnica de volume rendering (VR) donde se observa el tronco de la arteria pulmonar (TAP) y la rama pulmonar izquierda (RPI) pero no se visualiza la rama pulmonar derecha)

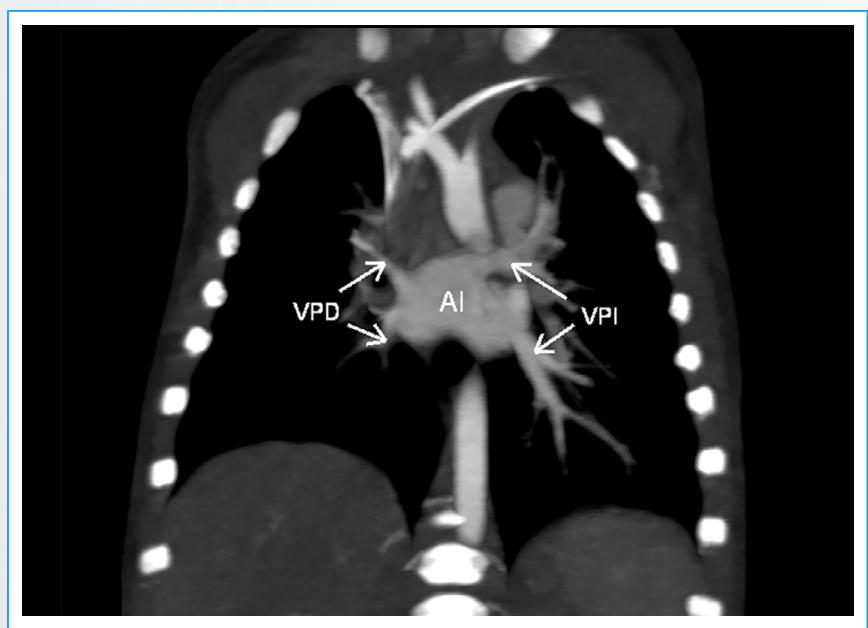

Figura 3. Reconstrucción coronal en MIP fina de $5 \mathrm{~mm}$ de grosor que muestra las venas pulmonares derechas (VPD) con un diámetro inferior respecto a las del lado izquierdo (VPI), desembocando todas ellas en la aurícula izquierda (Al) 


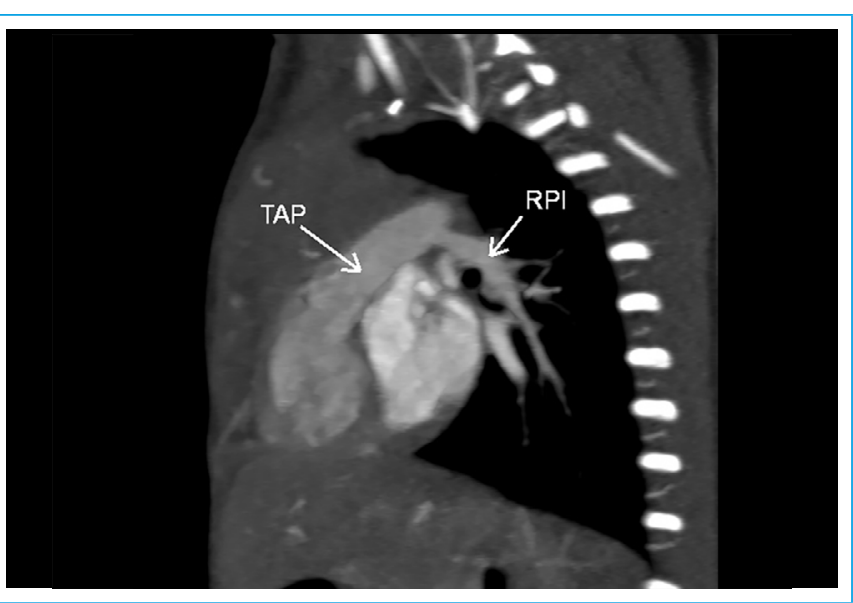

Figura 4. Reconstrucción sagital en MIP fina de $3 \mathrm{~mm}$ de grosor en el que se aprecia el origen normal de la rama pulmonar izquierda (RPI) en el tronco de la arteria pulmonar (TAP)

\section{Discusión}

La ausencia unilateral de la arteria pulmonar (UAPA) o agenesia de arteria pulmonar es un trastorno congénito poco frecuente, con una prevalencia en la población general desde 1:200.000 hasta 1:300.000(1), que puede aparecer de manera aislada pero normalmente se presenta asociado a otras anomalías congénitas como son la tetralogía de Fallot o comunicaciones septales. Otros defectos que también han sido asociados con una agenesia de arteria pulmonar son hipoplasia o ausencia del pulmón ipsilateral, coartación de la aorta, estenosis aórtica subvalvular o transposición de grandes vasos ${ }^{(2,3)}$.

El diagnóstico de una UAPA en período perinatal es raro dado que suele ser asintomático hasta la adolescencia o incluso la edad adulta y su detección en la infancia suele ser consecuencia de estudio por sobreinfecciones pulmonares en pacientes con bronquitis de repetición, hallazgo de hipoplasia pulmonar o hemoptisis debido a la alteración en la vascularización pulmonar que acompaña a esta anomalía. Sin embargo, la mayoría de los pacientes que no tienen asociada una anomalía cardíaca presentan síntomas leves o incluso ausentes y no suelen ser diagnosticados hasta la edad adulta ${ }^{(4)}$.

Los casos de ausencia de arteria pulmonar derecha doblan en número a los de la izquierda. No obstante, la agenesia de la arteria pulmonar izquierda suele ir asociada a malformaciones cardiovasculares más graves y, por eso, es importante su diagnóstico precoz y posterior tratamiento(5).

En niños menores de un año el síntoma predominante puede ser el distrés respiratorio asociado a hipertensión pulmonar (HTP), lo cual agrava el pronóstico, con el desarrollo posterior de insuficiencia cardíaca progresiva. Más allá del año de edad pueden presentar infecciones respiratorias recurrentes $y / 0$ hemoptisis y en la adolescencia o en la edad adulta una disminución de la capacidad pulmonar durante el esfuerzo y una limitación al ejercicio ${ }^{(5,6)}$.

El principal defecto embriológico es una involución del sexto arco aórtico proximal del lado afectado, con la consecuente ausencia de la arteria pulmonar proximal(5). Por otro lado, los vasos intrapulmonares y la porción distal de la arteria pulmonar afectada pueden tener un desarrollo normal debido a su origen embriológico diferente ${ }^{(7)}$. La agenesia de una rama pulmonar puede llevar asociada una ausencia total o parcial del pulmón ipsilateral. Cuando este está presente, aunque sea de manera subdesarrollada, la perfusión pulmonar es provista por colaterales sistémicas, principalmente por las arterias bronquiales, así como por las arterias intercostales a través de ramas transpleurales, la arteria mamaria interna, la subclavia o el tronco braquiocefálico ${ }^{(4)}$.

El diagnóstico de este tipo de patologías congénitas debe ser realizado mediante técnicas de imagen como la ecografía transtorácica, la tomografía computarizada o la resonancia magnética. A pesar de emplear radiaciones ionizantes, la angio TC es la técnica gold-standard para su diagnóstico definitivo por delante de otras como la angioresonancia magnética (angio RM) debido a su mayor rapidez, a su mayor disponibilidad en los servicios de diagnóstico por imagen y a la mejor resolución espacial de las imágenes obtenidas. Por otra parte, los avances en la técnica y en los equipos de TC permiten realizar estudios en neonatos usando dosis mínimas de radiación.

El tratamiento de esta patología suele ser conservador en la mayoría de los casos en los que la UAPA no va acompañada de malformaciones cardiovasculares graves, actuándose únicamente tras la aparición de los síntomas asociados $^{(8)}$. Así, la HTP puede ser tratada con fármacos antihipertensivos y la hemoptisis puede precisar de una embolización de las arterias colaterales, aunque la progresión de los síntomas en estos casos suele ser inevitable y de mal pronóstico.

\section{Conclusión}

La UAPA es un trastorno poco frecuente que puede hallarse de manera aislada o asociada a otras patologías cardiovasculares congénitas. El screening de cardiopatías congénitas que ya está establecido en todos los protocolos de revisión del niño sano es fundamental para el diagnóstico de cardiopatías potencialmente graves y frecuentemente asintomáticas durante este período.

Pese a que la ecocardiografía puede sugerir el diagnóstico, la técnica goldstandard para el diagnóstico definitivo en estos casos es la angio TC ya que permite, no sólo estudiar todo el árbol vascular pulmonar arterial y venoso, sino también la presencia y características del parénquima pulmonar.

\section{Ideas para recordar}

- La agenesia de arteria pulmonar es una patología congénita que, aunque suele estar asociada a otros defectos cardíacos congénitos, también puede hallarse de manera aislada.

- La mayoría de los pacientes que no tienen asociada una anomalía cardíaca no suelen ser diagnosticados hasta la edad adulta y su detección en período perinatal suele ser excepcional. Por ello, es importante hacer estudio de screening de cardiopatías congénitas en neonatos.

- Aunque la ecocardiografía transtorácica permite orientar el diagnóstico en muchos casos, el TC es una ayuda fundamental debido a su mayor resolución espacial y a la posibilidad de realizar reconstrucciones multiplanares y en 3D.

\section{Bibliografía}

1. Weldetsadik AY, Asfaw YM, Tekleab AM. Isolated absence of right pulmonary artery in a 4-year old child: a case report. Int Med Case Rep J. 2018; 11:297-301.

2. Espinosa $L$, Agarwal P. Adult presentation of right lung agenesis and left pulmonary artery sling. Acta Radiol. 2008; 49: 41-4.

3. Alison $\mathrm{M}$, Garel L, Bigras JL, et al. Unilateral absence of pulmonary artery in children: Bronchovascular anatomy, natural course and effect of treatment on lung growth. Pediatr Radiol. 2011; 41: 459-68. 
4. Bueno J, Flors L, Mejía M. Anomalías congénitas de las arterias pulmonares: espectro de hallazgos en tomografía computarizada. Radiología. 2017; 59 (3): 209-217.

5. Hayek H, Palomino J, Thammasitboon S. Right pulmonary artery agenesis presenting with uncontrolled asthma in an adult: a case report. J Med Case Rep. 2011; 5: 353.

6. Koga H, Hidaka T, Miyako K, et al. Age-related clinical characteristics of isolated congenital unilateral absence of a pulmonary artery. Pediatr Cardiol. 2010; 31: 1186-90
7. Castaner E, Gallardo X, Rimola J, et al. Congenital and acquired pulmonary artery anomalies in the adult: radiologic overview. Radiographics. 2006; 26 : 349-71.

8. Kruzliaka P, Syamasundarc RP, Novak M, Pechanova O, Kovacova G. Unilateral absence of pulmonary artery: Pathophysiology, symptoms, diagnosis and current treatment. Arch Cardiovasc Dis. 2013; 106: 448-54. 